\section{Nervous Mechanism in Tunicata}

DESPITE the contributions of Lorleberg1, Hilton ${ }^{2}$, Hecht ${ }^{3}$, Cate $^{4}$, Bacq $^{5}$ and Das ${ }^{6}$, the picture of tunicate nervous relations is still incomplete. Although the central nervous system consists of only one compact intersiphonal ganglion and the peripheral system of a few nerves emanating from the former, the extreme sensitivity of monascidians to touch, vibrations, etc., is astounding. A person walking about in a room where expanded Ciona, Ascidiella, or Styelopsis are lying in a jar on a table is enough to make some of the ascidians contract. Lorleberg ${ }^{1}$ stated that Styelopsis had free nerve terminations, whereas Das ${ }^{6}$ found in Herdmania an extensive nerve supply, not only to the organs lying inside the mantle, but also to receptor cells of the test as well. I have now found these nerve fibres, ramifying into the mantle, the test, and ending in the receptor organs, in Ciona, Ascidiella as well as Styelopsis. Free nerve terminations have been found in Styelopsis and Ascidiella and probably occur in all Tunicata.

The nerves arising from the intersiphonal ganglion have both afferent and efferent fibres in them; and as they branch and subdivide to supply the muscles and the receptors, the afferent and efferent fibrils are separated to form the peripheral nerves. It has also been established that, in crossed reflexes, nerve impulses pass directly from one siphon to the other by way of the ganglion. Removal of the ganglion not only prevents crossed reflexes but also affects the threshold of sensitivity and rate of recovery.

A complete account of the nervous mechanism in the Tunicata is being published elsewhere.

Department of Zoology,

S. M. DAS

University, Lucknow. June 30.

${ }^{1}$ Lorleberg, Z. wiss. Zool., 88, 212 (1907).

2 Hilton, Zool. Jahrb. Anat. Abt., 37, 113 (1913).

${ }^{3}$ Hecht, J. Exp. Zool. Philadelphia, 25, 229 (1918).

4 Cate, Arch. Meerlaud Sc. La Haye, 13, 45 (1928).

${ }^{5}$ Bacq, Bull. Acad. Belgique, Cl. Sci., (5), 20, 11 (1934).

- Das, J. Morph. Philadelphia, 59, (3), 589 (1936).

\section{Interpretation of Experiments on Metabolic Processes, using Isotopic Tracer Elements}

In two instances, the distribution of isotopic carbon in the product of a metabolic process has been used to infer that a symmetrical intermediate compound is not involved. Wood et al. ${ }^{1}$ showed that isotopic carbon, introduced as carbon dioxide together with pyruvate, led to the formation of ketoglutarate which contained isotopic carbon only in the carboxy group next to the keto group; on these grounds, they excluded citrate as an intermediate. Sbemin ${ }^{2}$ found that when glycine is formed from serine, containing isotopic nitrogen and isotopic carbon in its carboxy group, the relative abundance of nitrogen-15 and carbon-13 in the glycine was the same as in the serine; he argued that amino-malonic acid is therefore not an intermediate in this process.

These conclusions seem to arise from the fallacy that, because symmetrical products arising from the $d$ - or $l$-form of an optically active precursor cannot be distinguished, therefore the two identical groups of a symmetrical product formed from one optical antipode cannot be distinguished. On the contrary, it is possible that an asymmetric enzyme which attacks a symmetrical compound can distinguish between its identical groups. This power of distinction is illustrated for the case of amino-malonic acid in the accompanying formulæ. $a^{\prime}, b^{\prime}, c^{\prime}$ represent points in the enzyme which specifically combine with the groups $a, b, c$ of the substrate. Evidently, decarboxylation could occur at $a^{\prime}$ but not at $b^{\prime}$, or vice versa. The same conclusion follows if any three of the groups of the substrate specifically combine with the enzyme. If decarboxylation occurs only at $b^{\prime}$, then all the isotopic carbon in the carboxy group of serine will appear in the glycine. The case of the formation of ketoglutarate from citrate is exactly parallel.

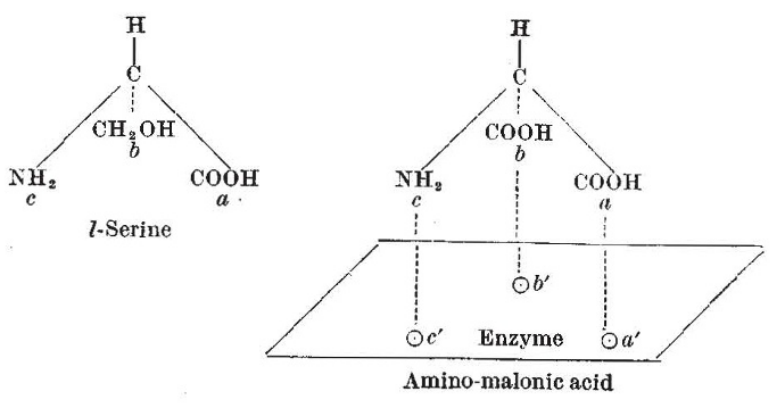

This argument depends on two conditions: $(a)$ that the sites $a^{\prime}$ and $b^{\prime}$ are catalytically different; $(b)$ that three-point combination occurs between the sym. metrical substrate and the enzyme; such combination is, of course, necessary wherever a single optical antipode is formed enzymatically from an inactive pre. cursor. Since neither is unlikely, it follows that the asymmetrical occurrence of isotope in a product cannot be taken as conclusive evidence against its arising from a symmotrical precursor.

\section{A. G. OGston}

Department of Biochemistry, Oxford.

July 12.

${ }^{1}$ Wood, Werkman, Hemingway and Nier, J. Biol. Chem., 139, 483 (1941).

2 Shemin, J. Biol. Chem., 162, 297 (1946).

\section{Cultivation of Trypanosoma cruzi in the Developing Chick Embryo}

ALтнолgh Trypanosoma cruzi, the causative agent of Chagas' disease in South America, has been successfully cultivated in the embryonic tissues of the chick, attempts at growing this parasite in the developing chick embryo have hitherto led to incon. clusive results. Roubaud and Romana ${ }^{1}$ inoculated $T$. cruzi into the chorio-allantoic membrane of chick embryos from (a) a rich culture; (b) drops of liquid excreta of Rhodinus prolixus containing metacyclic forms; and $(c)$ citrated blood of an infected guinea pig containing numerous large and active trypanosomes. The results were negative, and no infection was observed, except that in one embryo inoculated with the citrated blood a single trypanosome was found to have persisted on the membrane a week after inoculation. Rodhain and van den Berghe ${ }^{2}$ inoculated 